\title{
Fault diagnosis of rolling element bearings using artificial neural network
}

\author{
Saadi Laribi Souad ${ }^{1}$, Bendiabdellah Azzedine ${ }^{2}$, Samir Meradi ${ }^{3}$ \\ ${ }^{1}$ Department of Electrical Engineering, Laboratory of L2GEGI, University Ibn Khaldoun, Algeria \\ ${ }^{2}$ Department of Electrical Engineering, Laboratory of LDEE, \\ University of Sciences and Technology Mohamed Boudiaf, Algeria \\ ${ }^{3}$ Department of Electrical Engineering, National Polytechnic School, Algeria
}

\begin{tabular}{l}
\hline Article Info \\
\hline Article history: \\
Received Feb 13, 2019 \\
Revised Apr 28, 2020 \\
Accepted May 9, 2020 \\
\hline Keywords: \\
Bearing \\
Detection \\
Diagnosis \\
MLP neural network \\
Time-domain analysis \\
\hline
\end{tabular}

\begin{abstract}
Bearings are essential components in the most electrical equipment. Procedures for monitoring the condition of bearings must be developed to prevent unexpected failure of these components during operation to avoid costly consequences. In this paper, the design of a monitoring system for the detection of rolling element-bearings failure is proposed. The method for detecting and locating this type of fault is carried out using advanced intelligent techniques based on a perceptron multilayer artificial neural network (MLP-ANN); its database uses statistical indicators characterizing vibration signals. The effectiveness of the proposed method is illustrated using experimentally obtained bearing vibration data, and the results have shown good accuracy in detecting and locating defects.
\end{abstract}

Copyright $(2020$ Institute of Advanced Engineering and Science. All rights reserved.

Corresponding Author:

Saadi Laribi Souad,

Department of Electrical Engineering, Laboratory of L2GEGI,

University Ibn Khaldoun, Tiaret, Algeria.

Email: souad.laribi@univ-tiaret.dz

\section{INTRODUCTION}

The objective of condition-based maintenance is to detect failures of rotating machinery before a critical failure occurs. This maintenance approach has many advantages, as it avoids the need to dismantle the machine to check the condition of its components. In addition, the chances of detecting a defect before it becomes critical increase, thus avoiding a maximum of losses and failures of the machine. For these reasons, the automation of fault diagnosis in processes is indispensable. The industrial sector has aroused the interest of several researchers over the past decades.

Bearings in rotating machinery are vulnerable components and the presence of defects in the bearings can lead to the failure of these machines. The bearings generally consist of two rings, an inner and an outer, between which there is a set of balls or rotating rollers. Ball bearing defects are generally classified in the category of eccentricity defects and can be classified as a defect of the outer ring, defect of the inner ring and defect of balls [1].

Therefore, early identification of the seriousness of these bearing faults during operation can prevent machinery malfunction and failure. Defective bearings cause vibrations, therefore these vibration signals can be used to evaluate them. The first defect detection approaches consisted of the analysis of vibration signals extracted from the machine [2-5].

Diagnostic techniques for bearing failures can be classified into three approaches: time analysis based on statistical parameters [5, 6], frequency analysis [7, 8], and time-frequency analysis, such as the wavelet transform (WT) [9-11] and the Hilbert-Huang transform (HHT) [12]. Time-based diagnostics is not very effective when applied to incipient faults, or when the system is exposed to low loads, as described in [6]. 
Spectral analysis is the most classic approach to detecting failures in rotating machinery, and envelope analysis is the most popular method of diagnosing bearing failures. In a complex vibration signal from an industrial machine, envelope analysis can be used to extract periodic impacts such as those produced during bearing deterioration [6]. However, this classic tool is seriously affected by noise, especially in the early stages of defects. Another tool usually used to examine the frequency domain of signals is empirical mode decomposition (EMD), which allows to decompose a signal into different modes or IMFs (intrinsic mode function) $[13,14]$.

Due to the drawbacks of frequency analysis, it is necessary to find additional methods for analyzing the non-stationary of the signal. Time-frequency domain analysis is the most popular approach for the analysis of such signals; some can be cited such as the Wigner-file distribution (WVD) and the short-term fourier transform (STFT). These methods perform a one-dimensional signal transformation to a two-dimensional function; and can therefore provide true time-frequency representations of the signal, but each of the frequency-time analysis methods suffer from some problems. However, the use of these methods for identifying the nature of the defect and the estimation of its severity requires the knowledge of the expert who is not always available $[15,16]$. Today, thanks to the development of artificial intelligence techniques, this obstacle is overcome. These techniques are capable of managing a monitoring procedure of which they represent the expertise side and thus take the role designed for the expert.

In a diagnostic procedure, after the extraction of characteristics, an intelligent classification system is required. Several intelligent classification systems have been developed and used for monitoring and diagnosis, such as fuzzy classifiers, genetic algorithms, and support vector machines (SVMs) and artificial neural network (ANN) [17-19]. N. Talbi, et al., [19] proposed a robust bearing defect classification technique, which was used by the adaptive network reference information system (ANFIS). The results obtained by this technique gave a better accuracy of defect classification. In this context, we have tried to develop another approach for the detection and classification of bearing defects using again the advanced intelligent technique, but this time the technique is based on a perceptron multilayer artificial neural network (MLP-ANN). The method is proposed to combine vibration signal analysis and (MLP-ANN).

Several statistical indicators exist in the literature, more or less efficient and adequate to characterize a given signal. The indicators most sensitive to defects have been selected and used as inputs for (MLP-ANN). This technique is used for the detection and localization of bearing faults under different load conditions. The effectiveness of the proposed approach tests with experimental results.

\section{EXPERIMENTAL RESULTS}

The database used is a set of rolling vibration signals from "Case Western Reserve University Bearing Data Center, [20]. The database of normal or defective ball bearings is collected on a test bench consisting of a motor, a coupling and a generator. Defects in the form of dots of different diameters and depths have been created on the different parts of the bearings. As shown in Figure 1, the test bench consists mainly of a motor on the left side, a coupling "transducer / encoder" at the center, a dynamometer at the right side and the control circuits not visible in the Figure 1.

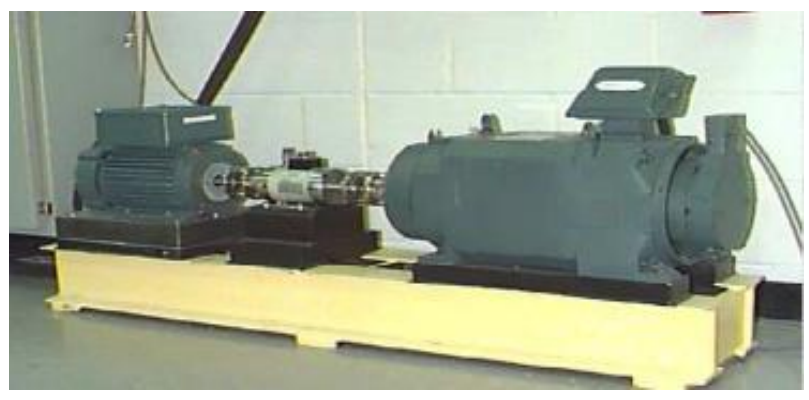

Figure 1. Experimental test-bed (Data bearing center)

\subsection{Identification of the bearings used}

The tested bearings support the motor shaft on both sides (drive side and fan side). Simple dot-shaped faults are created on the test bearings, using electro-discharge machining. Faults ranging from 0.007 inches to 0.028 inches in diameter are introduced separately in:

- The bearing inner ring; 
- The rolling element (the ball);

- The bearing outer ring.

All data files are in Matlab format (*.mat). Each file contains three data: vibration signals on both coupling and fan sides as well as the speed of rotation of the motor. The bearings used in our work are the side coupling bearings.

\subsection{Representation of time signals}

For the validation of the diagnostic procedure used, the acquisitions of time-domain vibration signals recorded on the coupling side for four load levels $(0,1,2,3 \mathrm{HP})$ and 12,000 points/second are for the following operating modes, see Figures 2 and 3: $1 \mathrm{mil}=0.001$ inches, $1 \mathrm{inch}=25.4 \mathrm{~mm}, 1 \mathrm{HP}=1 \mathrm{Horse}$ Power $=745.69$ Watt
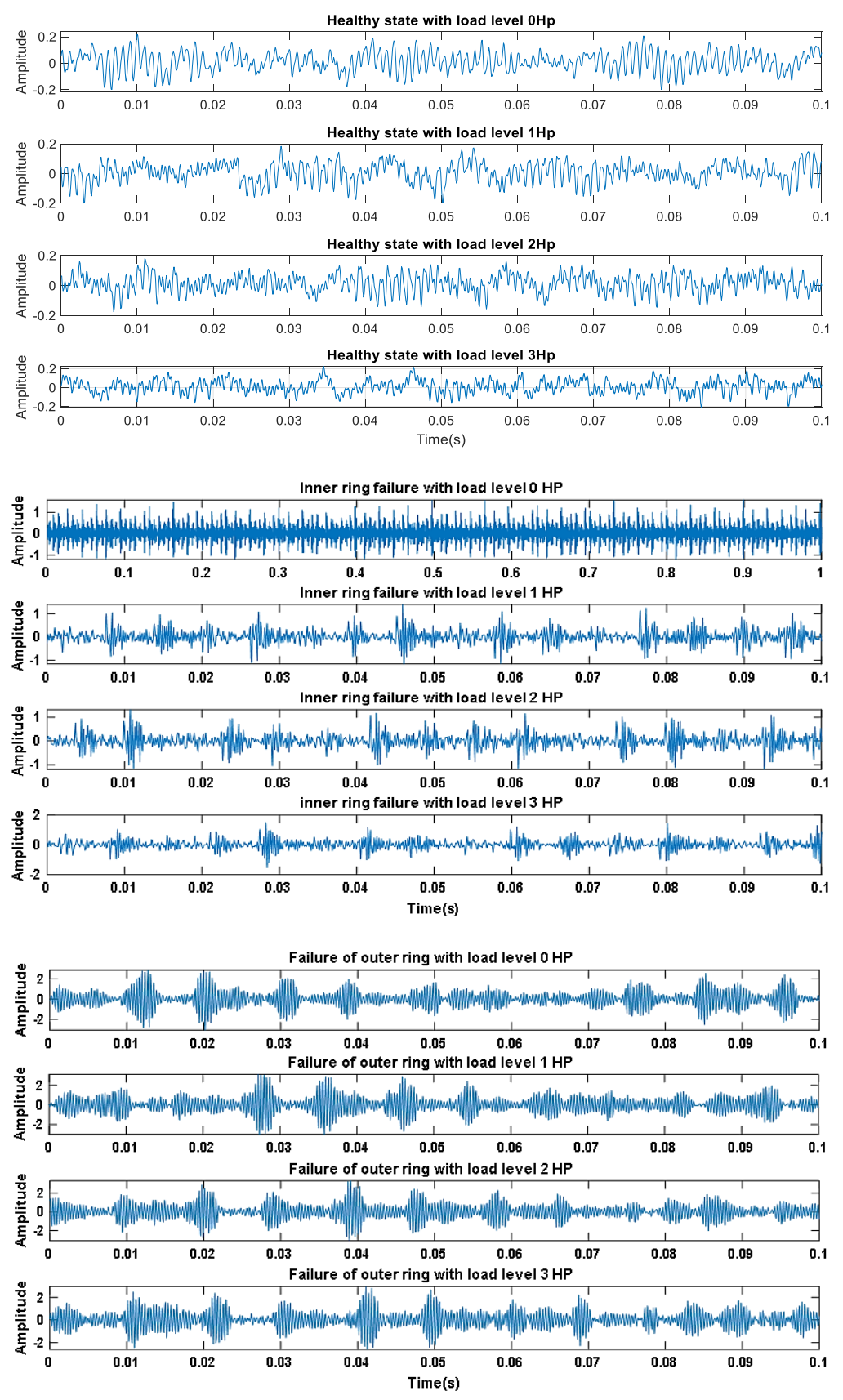

Figure 2. Vibration time signals for healthy and faulty operation for different resistive torques and defect diameters (0.007") 

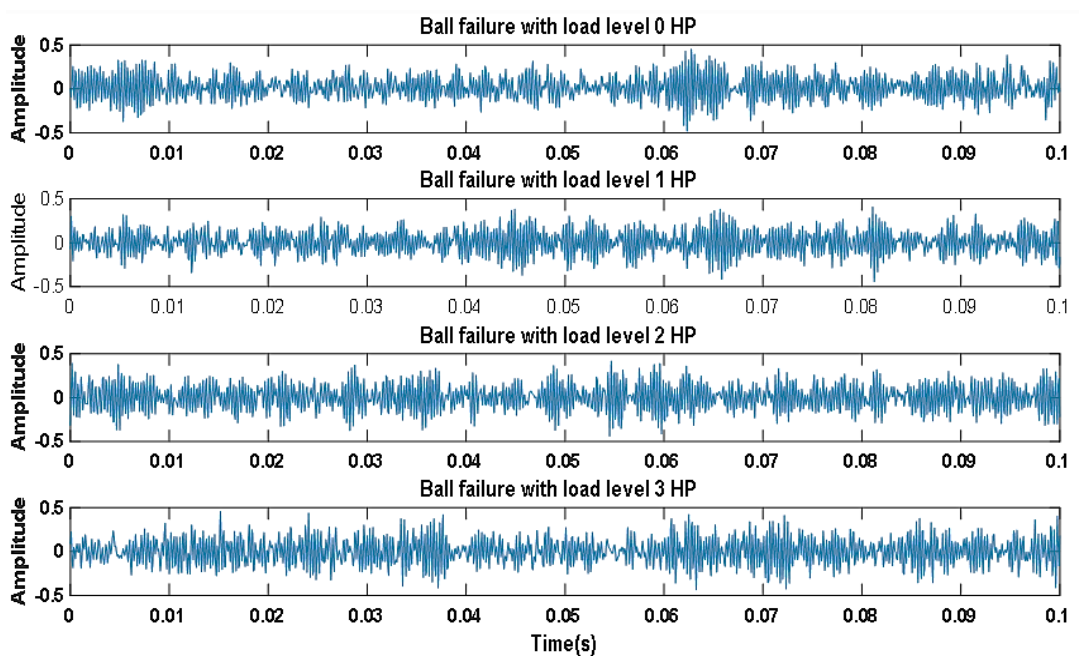

Figure 3. Vibration time signals for faulty operation for different resistive torques and defect diameters $(0.007 ")$

\section{APPLICATION OF ANN FOR DETECTION OF BEARING DEFECTS}

The amplitude of the shocks applied to the bearing depends directly on the diameter of the defect. The larger the defect, the greater the shock. The vibration signal, which is the response to the shock, is also directly related to the size of the defect. To characterize a vibration signal, several time descriptors exist, allowing having information by means of a unique value. The best known are the PEAK (maximum value), the RMS (root mean square) which represents the RMS value of the signal, the crest factor and the kurtosis. Table 1 summarizes the main conventional descriptors that we are going to use in our work.

Table 1. Temporal descriptors [21]

\begin{tabular}{ll}
\hline Temporal indicators & Expression \\
\hline Peak & peak $=\sup _{1 \leq \mathrm{K} \leq \mathrm{N}\left|\mathrm{a}_{\mathrm{k}}\right|}$ \\
Root Mean Square & $\mathrm{RMS}=\sqrt{\frac{1}{N}=\sum_{K=1}^{N} a^{2}{ }_{k}}$ \\
Kurtosis & Kurtosis $=\frac{\frac{1}{N}=\sum_{K=1}^{N}\left(a_{k}-\bar{a}\right)^{4}}{R M S^{4}}$ \\
Crest Factor & $\mathrm{IMF}=\frac{\text { peak }}{\frac{1}{N}=\sum_{K=1}^{N}\left|a_{k}\right|}$ \\
TALAF & $\mathrm{CF}=\frac{\text { peak }}{\mathrm{RMS}}$ \\
THIKAT & $\mathrm{TALAF}=\log \left[k_{u}+\frac{R M S}{R M S_{0}}\right]$ \\
\hline
\end{tabular}

In order to determine the type of existing bearing defect, a fault detection and diagnosis procedure are proposed using a combination of a statistical analysis technique based on vibration signal indicators and an intelligent technique based on artificial neural networks (ANN) [21-26]. Figure 4 shows a simplified flow chart of the proposed process.

The steps of construction and validation of neural networks are diving into three phases:

- Choice of network inputs

The learning base of the ANN is presented in the form of a file or a table (matrix). It is represented by classes of vectors, where each class represents a type of operation, and each vector is represented by the sampled values. In our case study, there are several vectors, which represent the four types of operation modes, and these represent four vectors for the different loads $(0,1,2,3 \mathrm{HP})$ and 12,000 points/second concerned. 
The sampled values chosen as inputs of the neural network are the statistical indicators of the vibration signals, their mathematical expressions are given in Table 1. Hence the number of inputs on this network is equal to 7 :

\section{[Peak RMS Ku IMF CF TALAF THIKAT $]^{T}$}

- Choice of network outputs

When a fault is detected, the network must indicate a binary number through its output, which corresponds to a type of fault; this is called the classification step. In this work, four types of faults are considered and are binary coded as shown in Table 2.

Table 2. Classification of types of rolling defects

\begin{tabular}{lc}
\hline Bearing condition & Codification/desired output \\
\hline Healthy state & 1000 \\
Fault in the inner ring & 0100 \\
Fault in the outer ring & 0010 \\
Fault in the ball & 0001 \\
\hline
\end{tabular}

- Choice of activation function

Since the outputs are binary and the inputs are real, the activation function is a sigmoid function as depicted in Figure 5. This figure illustrates the structure of the MLP-ANN neuron network used in our work.

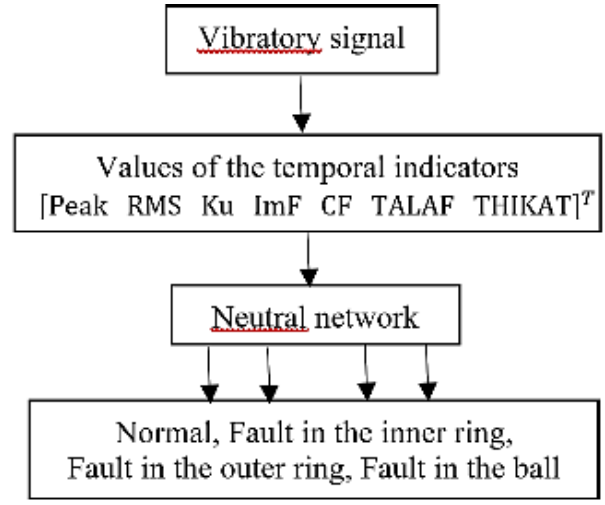

Figure 4. Classification scheme by RNA-based on values of the temporal indicators

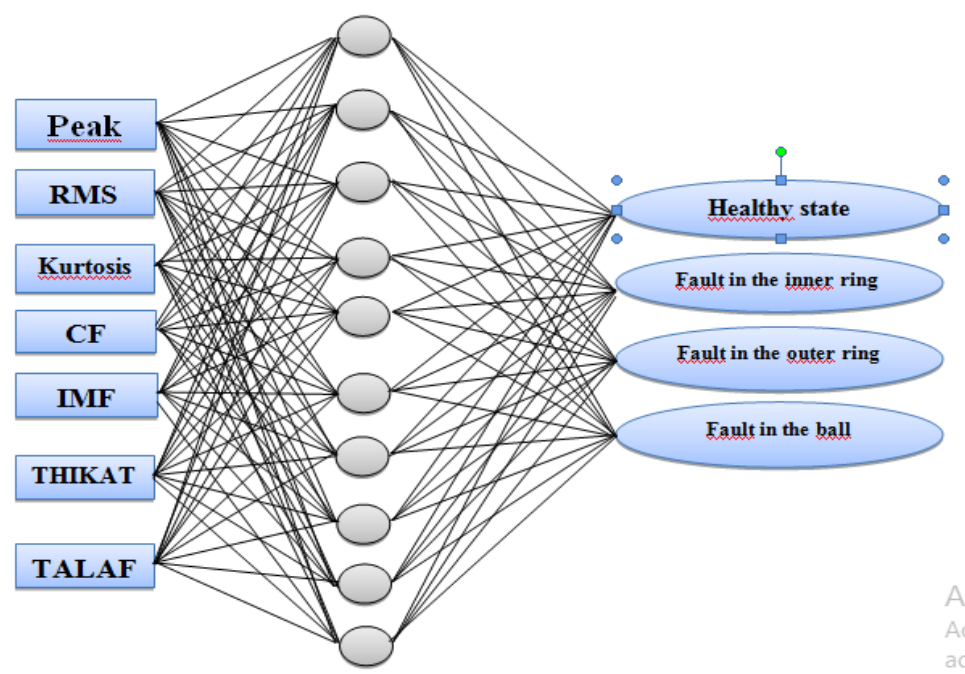

Figure 5. Neural network design 


\subsection{Results of learning}

The best learning performances obtained through a good choice of the ANN structure and after several learning tests. The resulting network is characterized by a simple architecture comprising an input layer (retina), an output layer for the decision and a hidden layer composed of ten neurons whose transfer function is of sigmoid type and of linear type for the neuron of the output layer as shown in Figure 6. The learning performance of the used ANN is evaluated through the mean squared error (MSE). For our case, the ANN reaches a value of 7.1192e-11 after 18 iterations as shown in Figure 7. From the results obtained in the test phase, can be seen that the outputs of the ANN follow almost exactly (with an error of 7.1192e -11) the desired pre-established outputs.

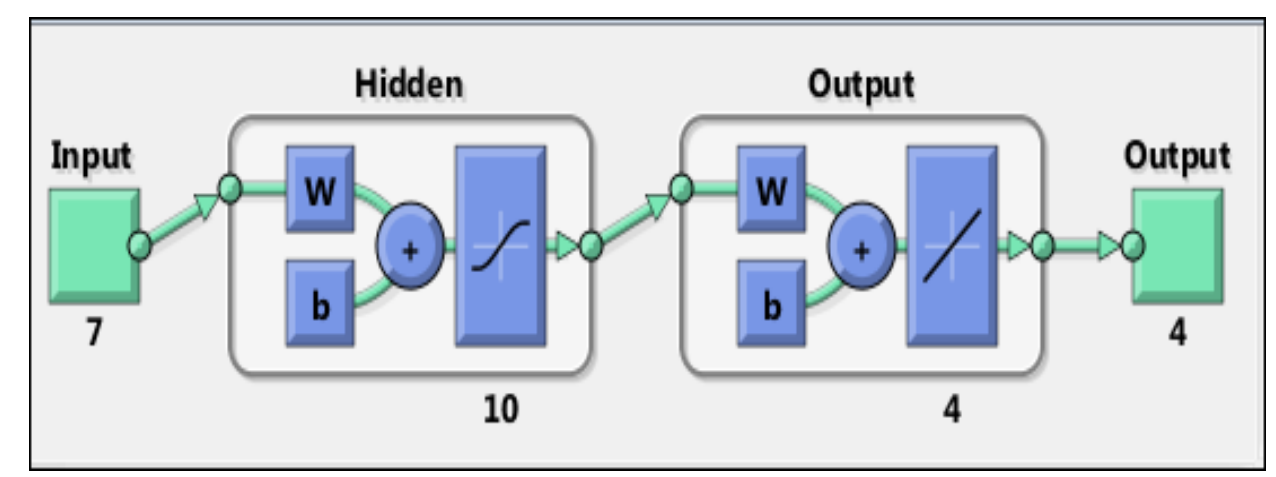

Figure 6. Building the ANN block

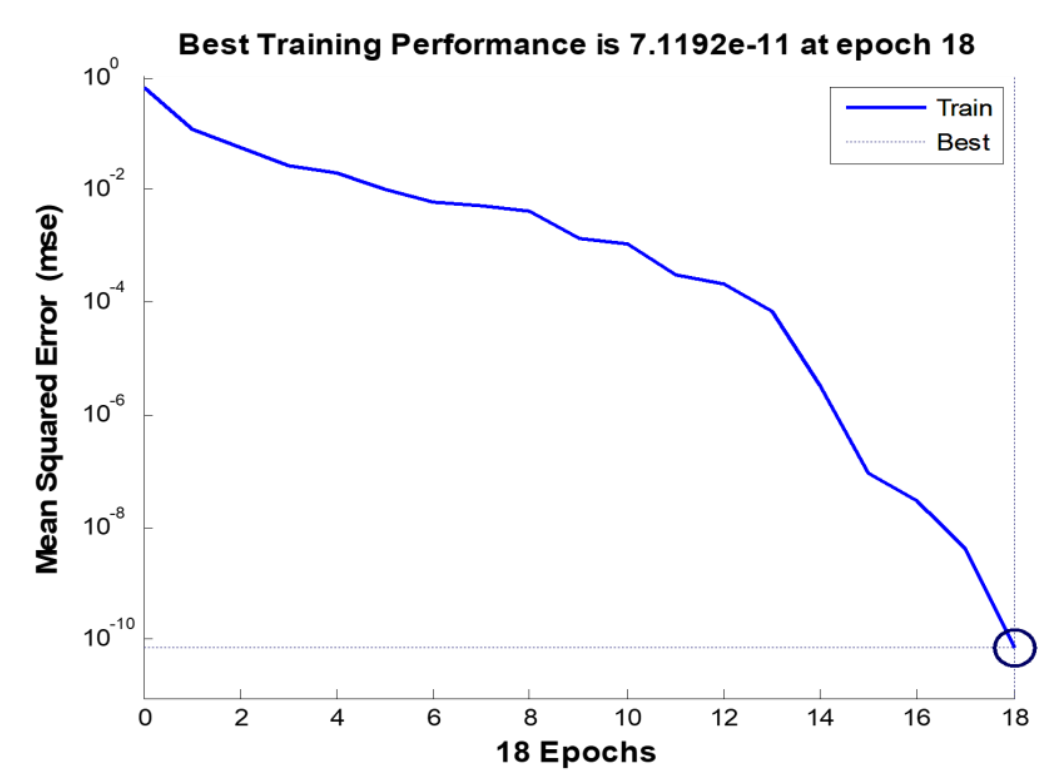

Figure 7. Performance of the neuron network

\subsection{Tests of the ANN for fault detection}

Once the ANN has built and its learning has achieved satisfactory performance, we move to the comparison stage between examples at the entrance of the network. In fact, these examples belong to two databases, the first being the learning base and the second being the test base on which one tests the ability of the network to recognize normal examples (not learned). The last operation makes it possible to estimate the generalization capacity of the ANN network. As for the network test on the examples that are not learned in the learning phase (new examples), their results are shown in Figure 8. From the results obtained in the test phase, it can be seen that the outputs of the ANN evolve according to the pre-established desired outputs for the different types of operation (healthy state and faulty state with bearing defects). 


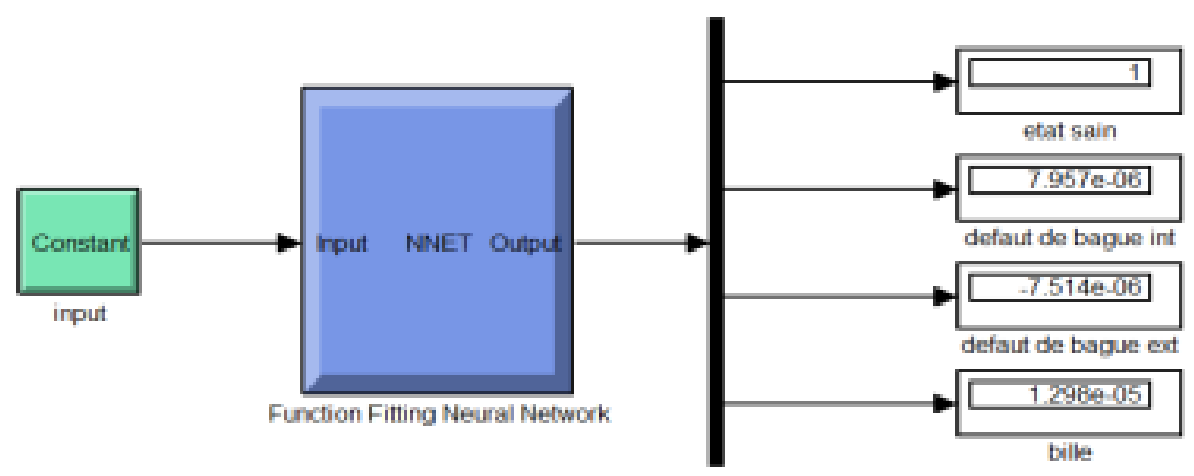

(a)

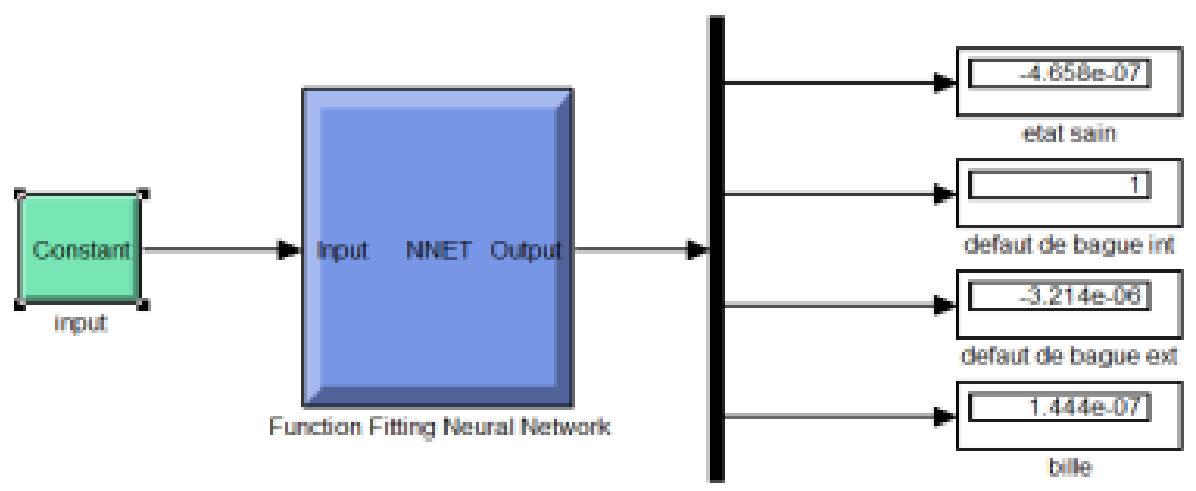

(b)

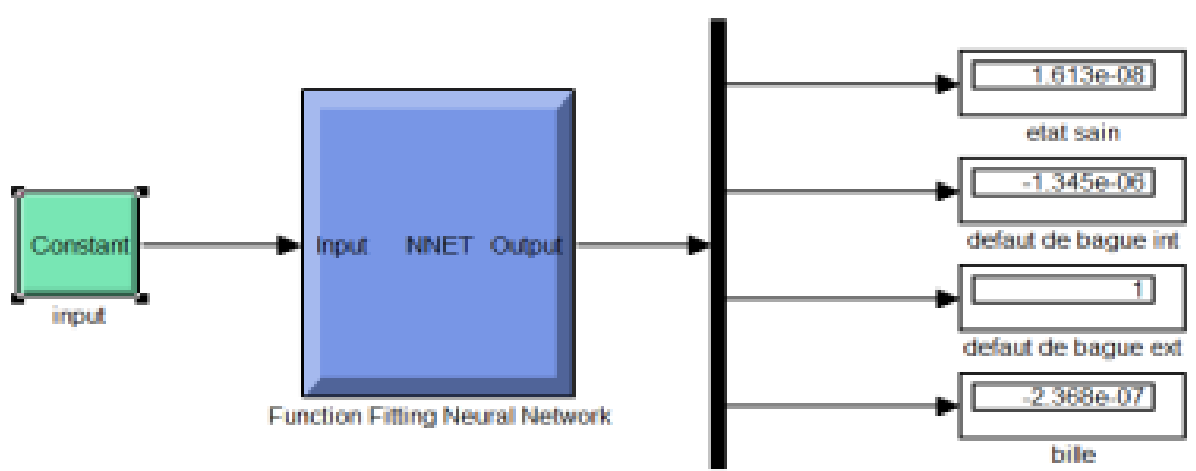

(c)

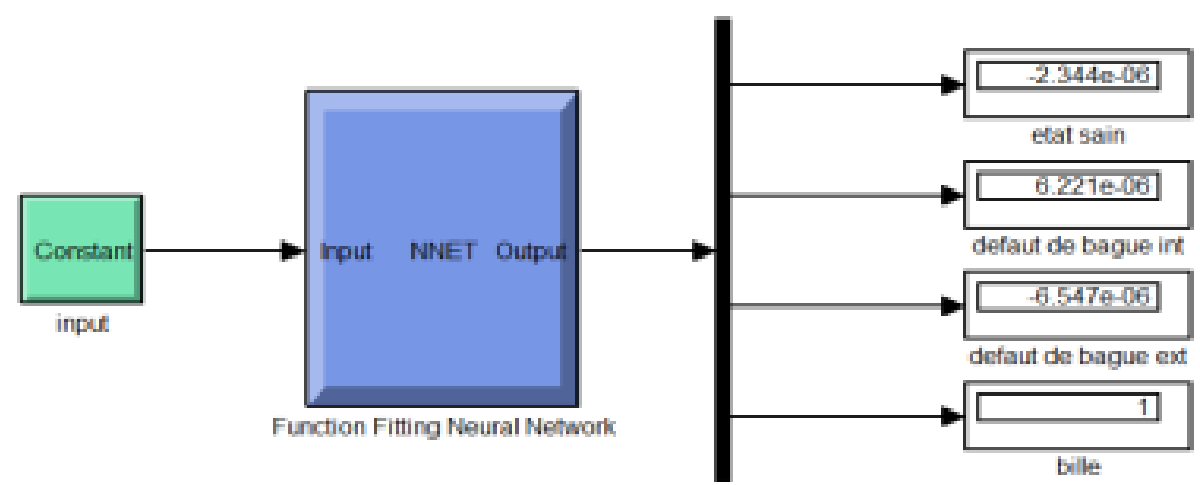

(d)

Figure 8. Outputs and errors of ANN learning, (a) Healthy machine: desired output 1000, (b) Machine with Fault in the inner ring: desired output 0100, (c) Machine with Fault in the outer ring: desired output 0010 and (d) Machine with Fault in the ball: desired output 0001 


\section{CONCLUSION}

This work is devoted to the proposal of the bearing defect classification using artificial neural networks (ANN). The resulting network is characterized by a simple architecture with an input layer (retina), an output layer for the decision and a hidden layer of 10 neurons, and graphical results that show the results of the training. It can be concluded that better learning performance achieves through a good choice of an ANN structure after several learning tests.

\section{REFERENCES}

[1] B. Muruganatham, et al., "Roller Element Bearing Fault Diagnosis Using Singular Spectrum Analysis," Mechanical Systems and Signal Processing, vol. 35, no. 1-2, pp. 150-166, 2013.

[2] S. Kulkarni and S. Wadkar., "Experimental Investigation for Distributed Defects in Ball Bearing Using Vibration Signature Analysis," Procedia Engineering, vol. 144, no. 2016, pp. 781-789, 2016.

[3] A. Pavan and P. Jayaswal, "Selection of Best Classification Algorithm for Fault Diagnosis of Bearing Using Vibration Signature Analysis," International Journal of Innovative Technology and Exploring Engineering, vol. 8, no. 5, pp. 538-546, 2019.

[4] A. Bouguerne, et al., "Optimisation of Vibration Data Using Particle Swarm Optimization Applied to Induction Machine Faults," International Conference on Renewable Energies, 2013.

[5] B. Muruganatham. et al., "Bearing Fault Detection and Classification Using ANC-Based Filtered Vibration Signal," International Conference on Communications and Cyber Physical Engineering, pp. 325-334, 2018.

[6] D. Didik, et al., "Fault Diagnosis of Roller Bearing Using Parameter Evaluation Techniques and Multi-Class Support Vector Machine," International Conference on Engineering, Science and Nanotechnology, 2016.

[7] A. Boudinar, et al., "Improved Stator Current Spectral Analysis Tchnique for Bearing Fault Diagnosis," 16th International Power Electronics and Motion Control Conference and Exposition, pp. 1228-1233, 2014.

[8] S. Djaballah and M. Kamel, "Detection and Diagnosis of Fault Bearing Using Wavelet Packet Transform and Neural Network," Article in Frattura Ed Integrità Strutturale, vol. 13, no. 49, pp. 291-301, 2019.

[9] L. C. José. et al., "Bearing Fault Diagnosis Based on Neural Network Classification and Wavelet Transform," Proceedings of the 6th WSEAS International Conference on Wavelet Analysis \& Multirate Systems, pp. 16-18, 2006.

[10] T. Narendiranath Babu, et al., "Journal Bearing Fault Detection Based on Daubechies Wavelet," Archives of Acoustics, vol. 42, no. 3, pp. 401-414, 2017.

[11] A. Kabla and K. Mokrani, "Bearing Fault Diagnosis Using Hilbert-Huang Transform (HHT) and Support Vector Machine (SVM)," Mechanics \& Industry, vol. 17, no. 3, 2016.

[12] W. Tsao, et al., "Resonant-Frequency Band Choice for Bearing Fault Diagnosis Based on EMD and Envelope Analysis, In Intelligent Control and Automation (WCICA)," 8th World Congress on Intelligent Control and Automation, pp. 1289-1294, 2010.

[13] J. Ben Ali, et al.,"Application of Empirical Mode Decomposition and Artificial Neural Network for Automatic Bearing Fault Diagnosis Based on Vibration Signals," Applied Acoustics, vol. 89, pp. 16-27, 2015.

[14] L. Hongmei, et al., "Rolling Bearing Fault Diagnosis Based on STFT-Deep Learning and Sound Signals," Shock and Vibration, vol. 2016, no. 2, pp. 1-12, 2016.

[15] R. R. Sharma, et al., "An Empirical Wavelet Transform-Based Approach for Cross-Terms-Free Wigner-Ville Distribution," Signal, Image and Video Processing, vol. 14, no. 2, pp. 249-256, 2020.

[16] S. Jagath, et al., "Early Detection and Classification of Bearing Faults using Support Vector Machine Algorithm," IEEE Workshop on Electrical Machines Design, Control and Diagnosis, pp. 250-255, 2017.

[17] A. Choug, et al., "Detection and Classification of Bearing Faults in Industrial Geared Motors Using Temporal Features and Adaptive Neuro-Fuzzy Inference System," Heliyon, vol. 5, no. 8, pp. 1-11, 2019.

[18] J. Lee, et al., "Fault Diagnosis of Induction Motor Using Convolutional Neural Network," Applied Sciences, vol. 9 , no. 15 , pp. 1-10, 2019.

[19] N. Talbi, et al., "Diagnosis of Bearing Defects by Anfis in The Induction Motor," Carpathian Journal of Electrical Engineering, vol. 13, no. 1, pp. 30-44, 2019.

[20] University C W R. "Bearing Data Center," ed Secondary, University C W R (United State: Case Western Reverse Univesity), 2016.

[21] P. Prof, et al., " Fault Detection in Rolling Element Bearing Using Vibration Based An,alysis," International Conference on Ideas, Impact and Innovation in Mechanical Engineering, vol. 5, no. 6, pp. 1666-1674, 2017.

[22] B. Narendiranath Babu, et al.,"Application of EMD ANN and DNN for Self-Aligning Bearing Fault Diagnosis," Archives of acoustics, vol. 43, no. 2, pp. 163-175, 2018.

[23] M. Talha, et al., "A Matlab and Simulink Based Three-Phase Inverter Fault Diagnosis Method Using Three-Dimensional Features," International Journal of Fuzzy Logic and Intelligent Systems, vol. 16, no. 3, pp. 173-180, 2016.

[24] E. Levent, "Bearing Fault Detection by One-Dimensional Convolutional Neural Networks," Mathematical Problems in Engineering, vol. 2017, pp. 1-9, 2017.

[25] C. Zhiqiang, at al., "Vibration-Based Gearbox Fault Diagnosis Using Deep Neural Networks," Journal of Vibroengineering, vol. 19, no. 4, pp. 2475-2496, 2017.

[26] B. Bessam. et al., "Detection of Broken Rotor Bar Faults in Induction Motor at Low Load Using Neural Network," ISA transactions, vol. 64, pp. 241-246, 2016. 